\title{
Baseline FDG PET-CT imaging is necessary for newly diagnosed inflammatory breast cancer patients: a narrative review
}

\author{
Miral M. Patel, Huong T. Le-Petross \\ Department of Radiology, The University of Texas MD Anderson Cancer Center, Houston, TX, USA \\ Contributions: (I) Conception and design: Both authors; (II) Administrative support: Both authors; (III) Provision of study materials or patients: None; \\ (IV) Collection and assembly of data: None; (V) Data analysis and interpretation: Both authors; (VI) Manuscript writing: Both authors; (VII) Final \\ approval of manuscript: Both authors. \\ Correspondence to: Miral M. Patel, MD. Assistant Professor, Department of Radiology, The University of Texas MD Anderson Cancer Center, 1515 \\ Holcombe, CPB5.3208, Houston, TX 77030, USA. Email: MPatel6@mdanderson.org.
}

\begin{abstract}
Objective: To review and discuss the rationale behind performing baseline 18-fluorodeoxyglucose positron emission tomography-computed tomography imaging for staging of inflammatory breast cancer patients.

Background: In the past three decades, the epidemiology of inflammatory breast cancer has resulted in separation of this entity from other breast cancer in staging and treatment. Advances in cancer imaging from 18-fluorodeoxyglucose positron emission tomography to 18-fluorodeoxyglucose positron emission tomography-computed tomography have now allowed for anatomic and functional correlation in evaluating extent of disease in cancer patients. Furthermore, studies throughout the past two decades have highlighted how 18-fluorodeoxyglucose positron emission tomography-computed tomography may play a role in staging inflammatory breast cancer patients given the uniqueness of this entity when compared to other breast cancers.

Methods: Narrative overview of the literature summarizing findings in the literature from searches in computerized databases and authoritative texts. The use of 18-fluorodeoxyglucose positron emission tomography-computed tomography with respect to regional nodal staging and distant metastasis detection in inflammatory breast cancer patients is reviewed. In addition, an overview of studies conducted to date comparing the sensitivity and specificity of 18-fluorodeoxyglucose positron emission tomography-computed tomography for baseline staging in inflammatory breast cancer patients is also provided. Therapeutic influences and effect on overall survival is discussed.

Conclusions: Baseline 18-fluorodeoxyglucose positron emission tomography-computed tomography allows for more optimal nodal staging, which has implications in prognosis and treatment of inflammatory breast cancer patients. It also allows for improved detection of metastasis on baseline presentation allowing therapy to potentially target these additional sites of disease.
\end{abstract}

Keywords: Inflammatory breast cancer (IBC); fluorodeoxyglucose positron emission tomography-computed tomography (FDG PET-CT); staging

Submitted Jun 28, 2021. Accepted for publication Oct 18, 2021.

doi: $10.21037 /$ cco-21-82

View this article at: https://dx.doi.org/10.21037/cco-21-82

\section{Introduction}

Inflammatory breast cancer (IBC) is a rare and very aggressive type of locally advanced breast cancer with poor prognosis (1). The frequency of IBC in the United States is approximately $1-5 \%$ of all diagnosed breast cancers (1) with newly diagnosed IBC patients being over $20 \%$ more likely to have distant metastasis at the time of diagnosis compared to $5 \%$ of non-IBC patients (2). It is characterized clinically by its rapid onset of skin erythema (peau d'orange) and breast edema. IBC tends to affect younger age groups in women and is often hormone receptor negative, thereby 
limiting treatment with hormonal therapies (1). IBC represents a distinct entity amongst all other breast cancers. The purpose of this paper is to review the current literature on the added benefit of FDG PET-CT evaluation in the initial staging of inflammatory breast cancer patients.

We present the following article in accordance with the Narrative Review reporting checklist (available at https:// dx.doi.org/10.21037/cco-21-82).

\section{Standard of practice}

Current imaging modalities available for detection and staging of all breast cancer, including IBC, include mammography (with or without tomosynthesis), contrastenhanced mammography, breast and nodal basin ultrasound, breast magnetic resonance imaging (MRI), molecular breast imaging (MBI), contrast enhanced computed tomography (CT) of the chest, abdomen and pelvis, 18-fluorodeoxyglucose positron emission tomographycomputed tomography (FDG PET-CT), and bone scan.

The current guideline for staging of all invasive breast cancer patients with radiological tests, published by the National Comprehensive Cancer Network (NCCN), recommends bilateral diagnostic mammography, ultrasound as necessary, and optional breast MRI with special consideration in patients who have mammographically occult malignancy (3). For patients with IBC, the recommendations remain similar with the addition of bone imaging (bone scan or sodium fluoride PET-CT) and contrast enhanced CT chest, abdomen and pelvis both as category $2 \mathrm{~B}$ recommendations. Category $2 \mathrm{~B}$ recommendation is defined as "based upon lower level evidence, there is NCCN consensus the intervention is appropriate" (3). FDG PET-CT is noted to be optional with the added note that it "may be performed at the same time as a diagnostic CT, is most helpful when standard staging studies are equivocal or suspicious, especially in the setting of locally advanced or metastatic disease and may also be helpful in identifying unsuspected regional nodal disease and/or distant metastasis in locally advanced breast cancer when used in addition to standard staging studies" (3).

\section{Objectives}

The purpose of this paper is to review the current literature on the added benefit of FDG PET-CT evaluation in the initial staging of inflammatory breast cancer patients. We present the following article in accordance with the narrative review reporting checklist.

Narrative search question: What is the basis for including FDG PET-CT in the baseline staging of IBC patients? Specifically, how does baseline FDG PET-CT compare to conventional staging of regional lymph nodes and distant metastasis? How do baseline FDG PET-CT findings affect treatment decisions?

\section{Methods}

A literature search was conducted using the electronic databases PubMed, GOOGLESCHOLAR, and Medline to identify publications on the topic of imaging examinations in staging of inflammatory breast cancer. Retrospective and prospective English-language papers published through the years 2000-2021 were included.

\section{PET/CT exam}

The use of FDG uptake in imaging patients with cancer has been well established. The advantage of FDG imaging is characterized by its ability to provide functional imaging based upon the knowledge that tumor cells tend to express transport proteins with greater affinity for glucose compared to normal cells, with greater influx of glucose into tumor cells $(4,5)$. Once in the tumor cells, FDG undergoes enzymatic changes resulting in trapping of FDG within the tumor cells causing greater uptake on imaging exams. FDG PET images can be evaluated semi-quantitatively for increased uptake with a standardized uptake value (SUV) $(4,6)$. The SUV reflects the degree of FDG uptake within a volume of interest (7). Traditionally, FDG PET imaging was performed as a strictly functional imaging exam; however, in the late 1990s, FDG PET-CT was introduced allowing coregistration of FDG PET images and CT images allowing for more accurate anatomic correlation $(4,8,9)$. Typical scanning protocols involve imaging beginning 60 minutes after intravenous FDG injection with CT and PET imaging performed independently and subsequently fused (7). Patients generally fast for at least 4 to 6 hours prior to the exam. Imaging extends from the vertex or base of the skull to the mid-thigh unless otherwise clinically indicated. In some cases, SUV cutoff values of 2-2.5 have been described for differentiating benign from malignant lesions $(4,10,11)$.

Previous studies evaluating the use of imaging examinations characterized by FDG uptake in breast cancer patients have demonstrated that within the breast, FDG uptake of breast cancer depends on the histologic and 
biologic characteristics of the tumor $(7,12-17)$. For example, studies have demonstrated milder uptake in ductal carcinoma in situ and invasive lobular carcinoma compared to invasive ductal carcinoma, and higher uptake with grade 3 tumors compared to grade 1 and 2 tumors (7,12-18). Lack of FDG uptake in the breast does not exclude the presence of breast malignancy, especially noninvasive malignancy or welldifferentiated primary breast cancers such as DCIS, tubular carcinoma and invasive lobular carcinoma $(4,10,17,19,20)$. And while FDG PET-CT has demonstrated 96-100\% sensitivity for the diagnosis of the primary breast tumor in IBC patients, it has also demonstrated false positive findings particularly in cases of mastitis where SUV values were elevated and unable to reliably differentiate between malignancy and inflammation $(21,22)$. In addition, limited spatial resolution also limits detection of small lesions. In a retrospective review performed by Carkaci et al., most false negative findings on FDG PET-CT involved small lesions $<1.3 \mathrm{~cm}$ (19). Therefore, FDG PET-CT is not routinely used in staging work-up of stage 1 or early stage 2 breast cancer patients (19). As such, the existing dedicated breast imaging examinations are utilized to evaluate local disease in the breast, however studies have shown a role for FDG PETCT in evaluating locoregional disease, specifically within the nodal basins and distant metastasis.

\section{Regional nodal staging}

Axillary nodal status is an important prognostic factor in IBC as it is a predictor of survival outcome (23). Axillary nodal metastasis is associated with shorter disease-free and overall survival compared to patients without nodal disease (24-26). Axillary nodal disease has been reported in $55-85 \%$ of IBC patients at initial staging (27). Preoperative radiologic staging is routinely performed using ultrasound. In 2009, Alberini et al. published the largest prospective study at the time evaluating FDG PET-CT in the staging and prognosis of patients with suspected IBC which highlighted advantages of FDG PET-CT in regional nodal staging (21). In the study's population of 59 IBC patients, $12 \%$ of the patients were found to have axillary nodal metastasis demonstrated by PET-CT which was not suspected by clinical examination and an additional $56 \%$ of patients were noted to have extra-axillary lymph node metastasis diagnosed by PET-CT (only $5 / 33$ patients with extra-axillary lymph node metastasis were detected by clinical examination and confirmed with FNA upon detection) (21). Extra-axillary was defined as retropectoral, infraclavicular, supraclavicular and internal mammary nodal basins/regions (21).

A retrospective study published in the same year by Carkaci et al. demonstrated high sensitivity of FDG PETCT in diagnosing regional nodal metastasis with $37 / 41$ (90\%) of ipsilateral axillary metastasis and 18/41 (44\%) of subpectoral metastasis detected on FDG PET-CT (19). In addition, $22 \%$ of the patients had internal mammary and $15 \%$ had supraclavicular nodal disease (19). Given the variability of the appearance of lymph nodes on sonography and CT examinations, FDG PET-CT proves invaluable in providing functional evaluation of the regional nodal basins in IBC patients. A subsequent 2012 retrospective study by Carkaci et al. found that evaluating the SUVmax of regional lymph nodes on FDG PET-CT may help quantitatively differentiate benign from malignant nodes in IBC patients with a SUV cutoff value of 2.0 demonstrating a sensitivity of $89 \%$ and specificity of $99 \%$ in diagnosing metastatic disease for this cohort (24).

Although regional nodal staging is most commonly done by ultrasound; there is great variation amongst institutions on the evaluated regions and management. While some practices may evaluate the ipsilateral axillary level I region, others may evaluate bilateral regional nodal basins on baseline ultrasound staging of IBC patients. Additionally, regardless of the nodal basins evaluated, inter-operator variability may exist with ultrasound examination. As such, FDG PET-CT provides advantages by standardizing staging in patients who may have extra-axillary or contralateral nodal metastasis which may otherwise be undetected. It is important to note that as yet, FDG PET-CT has not been able to completely replace sonographic staging as small axillary metastasis, including micrometastases may be missed on FDG PET-CT due to the limited spatial resolution $(7,19)$. However, as uptake also relies on FDG avidity and other non-imaging related parameters (such as respiratory motion), in certain favorable conditions, smaller $(<1 \mathrm{~cm})$ lesions may still be detected $(7)$.

\section{Distant metastasis}

IBC patients presenting with distant metastasis are treated with primary systemic therapy to achieve local response and subsequently evaluated for potential surgery and radiation therapy (28). Identifying metastatic disease may lead to local treatment of the metastatic lesion or changes in systemic therapy $(29,30)$. Therefore, optimizing treatment for IBC patients requires identification of distant metastasis at 
baseline staging.

In the 2009 prospective study by Alberini et al., FDG PET-CT suggested distant metastasis in $31 \%$ of patients (18/59 patients) with only 6 of them demonstrated by routine workup (21). Sites of distant metastasis included the mediastinum, bone, liver, lung, contralateral axilla, peritoneum/ovary, cervical nodes and contralateral breast (21). This study was further corroborated by the 2009 retrospective review by Carkaci et al. of 41 IBC patients who underwent staging FDG PET-CT which found $20 / 41$ patients $(49 \%)$ to have distant metastasis on initial staging FDG PET-CT (19). The most common sites of metastasis were found to be mediastinal lymph nodes (24\%), bone (22\%), and liver (15\%) (19). Contralateral nodal metastasis was also demonstrated in $17 \%$ of patients (19). Less common sites of metastasis included pulmonary metastasis (5\%) and abdominal nodal metastasis (7\%) (19). Additionally, in this study, 7/41 (17\%) of the patients were not suspected of having metastasis clinically or on baseline imaging (19).

Similar findings were noted in a subsequent 2013 prospective study by Groheux et al. of 117 patients comparing FDG PET-CT performance with conventional work-up (bone scan, chest radiograph/chest CT, abdominal ultrasound/CT abdomen) in patients with locally advanced or inflammatory breast cancer (31). Overall, FDG PETCT was noted to change the stage in $52 \%$ of patients with the stage modified more frequently $(46 \%)$ in patients with IBC compared to noninflammatory locally advanced breast cancer $(33 \%)$, though this was not found to be statistically significant (31). In this same study, all patients with osseous metastasis on bone scan were also positive on FDG PETCT with FDG PET-CT also finding an additional 7 cases of osseous metastasis seen solely on FDG PET-CT (31). Additionally, while prior studies have demonstrated lower SUV values in osteoblastic metastasis thereby limiting evaluation on traditional FDG PET studies without anatomic correlation, as the blastic features are visible on traditional CT, the hybrid FDG PET-CT scan can outperform bone scan (31).

\section{Comparative analysis}

Osseous metastasis has been traditionally described as the most common site of distant disease in breast cancer (32). These metastatic lesions may be lytic, blastic, or a combination of both. Several studies have demonstrated PET/ $\mathrm{CT}$ to be superior to bone scan in detecting lytic metastasis
(33-40) (Figure 1).

In a 2008 prospective study by Fuster et al., preoperative staging of 60 patients with large primary breast cancer with FDG PET-CT compared to conventional imaging procedures (chest CT, liver ultrasound, and bone scan) demonstrated the sensitivity and specificity for FDG PETCT in detecting axillary nodal metastasis to be $70 \%$ and $100 \%$, respectively (41). In the same study, the sensitivity and specificity of FDG PET-CT in detecting distant metastasis was $100 \%$ and $98 \%$ respectively, compared to sensitivity of $60 \%$ and specificity of $83 \%$ with conventional imaging, with with FDG PET-CT leading to a change in initial staging of $42 \%$ of patients (41). It is important to note that this study was not solely conducted on IBC patients and did not utilize CT abdomen in evaluating for abdominal metastases. However, given the relatively higher percentages of regional nodal metastasis and distant metastasis present in IBC patients, the sensitivity of FDG PET-CT in IBC patients is hypothesized to be higher (Figure 2).

In a subsequent retrospective study of 225 patients with primary breast cancer by Niikura $e t a l$. in 2011, even when eliminating patients with stage IV disease, the sensitivity and specificity of FDG PET-CT compared to conventional staging modalities (contrast enhanced CT, US, radiography and bone scan) was $96 \%$ and $91 \%$, respectively compared to $84 \%$ and $67 \%$, respectively (42). In addition, the sensitivity and specificity of FDG PET-CT compared to bone scan for detecting osseous metastasis was $98 \%$ and $96 \%$, respectively compared to $76 \%$ and $86 \%$ (42). With respect to hepatic metastasis, FDG PET-CT and abdominal CT performed similarly with FDG PET-CT demonstrating a sensitivity and specificity of $100 \%$ and $99 \%$, respectively, compared to $100 \%$ and $95 \%$, respectively, for abdominal CT (42).

\section{Therapeutic influences and survival}

Radiation therapy plays a key role in treatment of IBC as patients diagnosed with IBC without evidence of distant metastasis generally receive systemic chemotherapy followed by mastectomy with axillary node dissection and adjuvant radiation therapy (23). It is important to adequately stage IBC patients on baseline imaging to identify involved regional nodal basins so post mastectomy radiation therapy can treat surgically resected and unresected nodal basins $(23,43)$. In a retrospective study of 62 patients by Walker et al. in 2012, findings of FDG PET-CT led to changes in post mastectomy radiation therapy for IBC patients in $17.7 \%$ of patients (44). Potential changes to standard post 

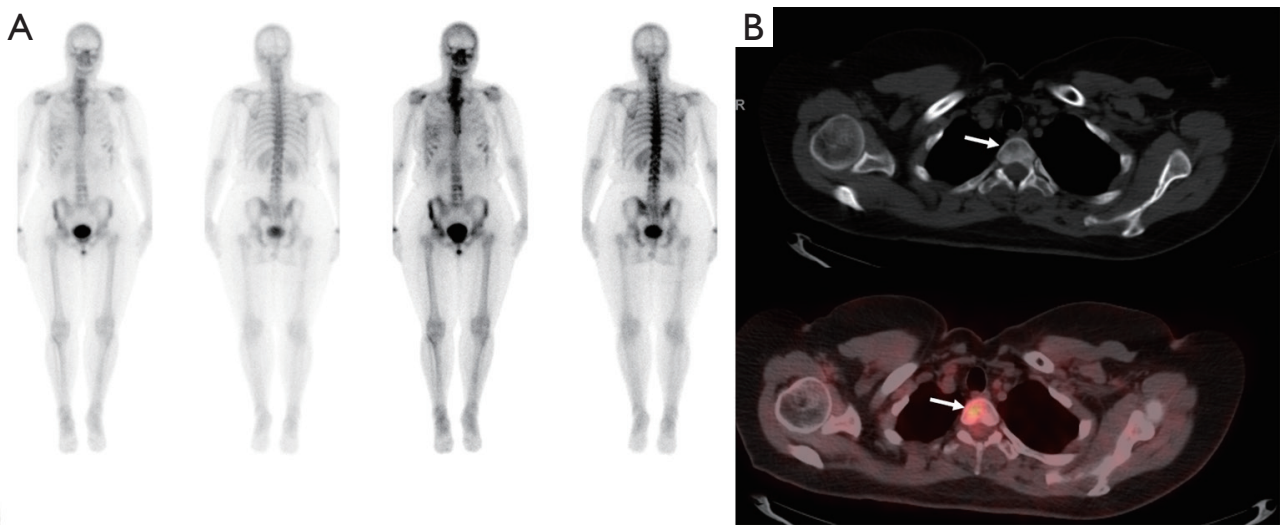

Figure 1 Supplemental case of a 54-year-old female with newly diagnosed right inflammatory breast cancer. (A) Bone scan performed at the time of diagnosis demonstrates focus of increased activity in the right $8^{\text {th }}$ rib and increased uptake in the right sacroiliac joint/iliac crest with no uptake in the spine. The patient subsequently underwent CT chest and abdomen with contrast (not pictured) demonstrating no evidence of osseous metastasis. (B) FDG PET-CT demonstrates focal lytic T2 vertebral body abnormality with increased FDG uptake (arrows) suspicious for metastasis which was confirmed on subsequent MRI thoracic spine with contrast.
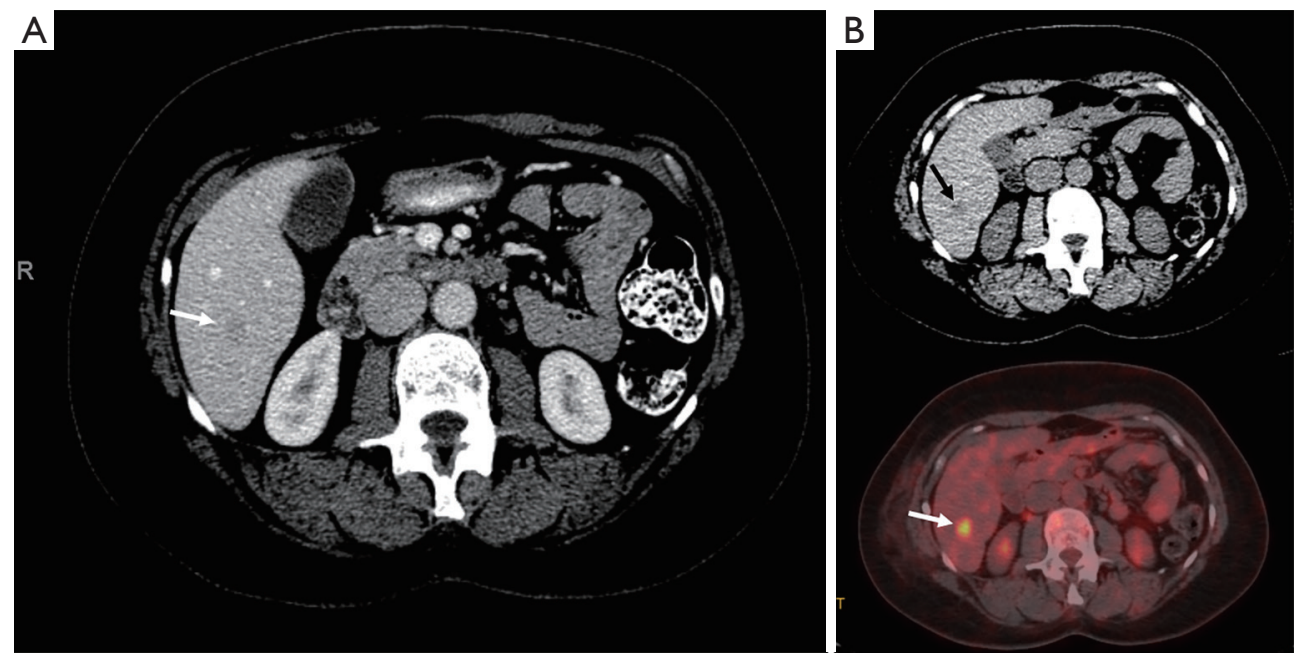

Figure 2 Supplemental case of a 54-year-old female with newly diagnosed right inflammatory breast cancer. (A) CT chest, abdomen and pelvis with contrast noted a subtle hypoattenuating lesion (arrow) in the posterior right hepatic lobe only seen on one slice, difficult to characterize. Additional correlation with MRI was recommended. (B) FDG PET-CT demonstrates the same focal hypoattenuating lesion in the posterior right hepatic lobe (black arrow) with associated FDG uptake (white arrow), maximum SUV 7.7, consistent with metastasis.

mastectomy radiation therapy may include modification to the radiation field design and radiation dose prescription (44). This retrospective study dedicated to IBC patients also found that, similar to the retrospective comparative analysis studies described above, FDG PET-CT found new areas of disease in $44 \%$ of patients which were not appreciated by routine imaging (44). In additional to nodal involvement, the extent of pretreatment skin involvement plays a role in determining post mastectomy radiation treatment (23). As IBC is noted to infiltrate the dermal lymphatics, it can present with a high risk of local recurrence (23). Baseline pre-treatment imaging of skin involvement may also play an important role in differentiating from post-radiation therapy skin thickening.

Thus far, survival benefits of changes in radiation plans due to FDG PET-CT in IBC patients has not been demonstrated. However, previous studies have 
demonstrated that the use of FDG PET-CT in IBC patients showed improved relapse-free survival (45).

\section{Limitations}

One limitation of all studies performed to date is that not every site of presumed metastasis detected on FDG PETCT was biopsy proven. Corroboration with pathology and additional imaging modalities, for example MRI if indicated, was attempted. It is important to note that even in a prospective trial, it may simply not be feasible to biopsy every possible metastatic site detected on FDG PETCT. An additional limitation is in performing comparative analysis between FDG PET-CT and baseline staging, there is institutional and provider variation on what baseline exams were performed. While some patients may have received CT chest and a hepatic ultrasound, others may have received CT chest, abdomen, and pelvis.

Limitations of FDG PET-CT in evaluation for metastasis must also be noted. One limitation of FDG PET$\mathrm{CT}$ is brain metastasis due to the baseline high uptake in the brain (31). In such situations, dedicated brain imaging with CT or MRI is recommended. An additional limitation of FDG PET-CT is evaluation of pulmonary metastasis given lack of sensitivity for small nodules especially considering respiratory motion and partial volume effects (31).

\section{Future research}

Thus far, the literature has demonstrated value in adding baseline staging FDG PET-CT for IBC patients, particularly in the realm of nodal staging, distant metastasis, and affecting post mastectomy radiation therapy. Prospective comparative studies evaluating the performance of current baseline imaging examinations to include contrast enhanced CT chest, abdomen and pelvis compared to FDG PET-CT specifically in IBC patients have yet to be performed. Further cost analysis in such a study may ultimately lead to changes in staging recommendations. From this, it may even be of added benefit to consider starting with FDG PETCT in patients already diagnosed with IBC prior to additional mammographic or sonographic evaluation to reduce imaging time and further reduce cost of imaging examinations and shorten the time from diagnosis to treatment. Further evolution to include contrast enhanced FDG PET-CT and PET/MR may have added value in the staging of IBC patients. Ultimately, prospective studies assessing the use of baseline FDG PET-CT and the effect on overall survival would be highly beneficial.

\section{Acknowledgments}

Funding: This work was supported by National Institutes of Health and National Cancer Institute Cancer Center Award Grant (P30CA016672).

\section{Footnote}

Provenance and Peer Review: This article was commissioned by the Guest Editors (Naoto Ueno and Angela Alexander) for the series "Inflammatory Breast Cancer" published in Chinese Clinical Oncology. The article has undergone external peer review.

Reporting Checklist: The authors have completed the Narrative Review reporting checklist. Available at https:// dx.doi.org/10.21037/cco-21-82

Conflicts of Interest: Both authors have completed the ICMJE uniform disclosure form (available at https://dx.doi. org/10.21037/cco-21-82). The series "Inflammatory Breast Cancer" was commissioned by the editorial office without any funding or sponsorship. The authors have no other conflicts of interest to declare.

Ethical Statement: The authors are accountable for all aspects of the work in ensuring that questions related to the accuracy or integrity of any part of the work are appropriately investigated and resolved.

Open Access Statement: This is an Open Access article distributed in accordance with the Creative Commons Attribution-NonCommercial-NoDerivs 4.0 International License (CC BY-NC-ND 4.0), which permits the noncommercial replication and distribution of the article with the strict proviso that no changes or edits are made and the original work is properly cited (including links to both the formal publication through the relevant DOI and the license). See: https://creativecommons.org/licenses/by-nc-nd/4.0/.

\section{References}

1. National Institutes of Health National Cancer Institute. Inflammatory breast cancer [Internet]. 6 Jan 2016 [cited 8 April 2021]. Available online: https://www.cancer.gov/ 
types/breast/ibc-fact-sheet\#: :text=Inflammatory\%20 breast $\% 20$ cancer\%20is\%20rare\%2C\%20accounting\%20 for $\% 201$, the $\% 20$ breast $\% 20$ and $\% 20$ then $\% 20$ spread $\% 20$ beyond $\% 20$ the $\% 20$ ducts.

2. Levine PH, Steinhorn SC, Ries LG, et al. Inflammatory breast cancer: the experience of the surveillance, epidemiology, and end results (SEER) program. J Natl Cancer Inst 1985;74:291-7.

3. National Comprehensive Cancer Network®. NCCN clinical practice guidelines in oncology: Breast cancer version 5.2020 [Internet]. 2020 [cited 8 April 2021]. Available online: https://www2.tri-kobe.org/nccn/guideline/ breast/english/breast.pdf.

4. Lim HS, Yoon W, Chung TW, et al. FDG PET/CT for the detection and evaluation of breast diseases: usefulness and limitations. Radiographics 2007;27 Suppl 1:S197-213.

5. Wahl RL. Targeting glucose transporters for tumor imaging: "sweet" idea, "sour" result. J Nucl Med 1996;37:1038-41.

6. Pauwels EK, Ribeiro MJ, Stoot JH, et al. FDG accumulation and tumor biology. Nucl Med Biol 1998;25:317-22.

7. Groheux D, Espié M, Giacchetti S, et al. Performance of FDG PET/CT in the clinical management of breast cancer. Radiology 2013;266:388-405.

8. Goerres GW, Von Schulthess GK, Hany TF. Positron emission tomography and PET CT of the head and neck: FDG uptake in normal anatomy, in benign lesions, and in changes resulting from treatment. AJR Am J Roentgenol 2002;179:1337-43.

9. Kluetz PG, Meltzer CC, Villemagne VL, et al. Combined PET/CT Imaging in Oncology. Impact on Patient Management. Clin Positron Imaging 2000;3:223-30.

10. Avril N, Dose J, Jänicke F, et al. Metabolic characterization of breast tumors with positron emission tomography using F-18 fluorodeoxyglucose. J Clin Oncol 1996;14:1848-57.

11. Adler LP, Crowe JP, al-Kaisi NK, et al. Evaluation of breast masses and axillary lymph nodes with F-18 2-deoxy-2fluoro-D-glucose PET. Radiology 1993;187:743-50.

12. Bos R, van Der Hoeven JJ, van Der Wall E, et al. Biologic correlates of (18)fluorodeoxyglucose uptake in human breast cancer measured by positron emission tomography. J Clin Oncol 2002;20:379-87.

13. Gil-Rendo A, Martínez-Regueira F, Zornoza G, et al. Association between 18Ffluorodeoxyglucose uptake and prognostic parameters in breast cancer. Br J Surg 2009;96:166-70.
14. Buck A, Schirrmeister H, Kühn T, et al. FDG uptake in breast cancer: correlation with biological and clinical prognostic parameters. Eur J Nucl Med Mol Imaging 2002;29:1317-23.

15. Avril N, Menzel M, Dose J, et al. Glucose metabolism of breast cancer assessed by 18F-FDG PET: histologic and immunohistochemical tissue analysis. J Nucl Med 2001;42:9-16.

16. Crippa F, Seregni E, Agresti R, et al. Association between 18Ffluorodeoxyglucose uptake and postoperative histopathology, hormone receptor status, thymidine labelling index and p53 in primary breast cancer: a preliminary observation. Eur J Nucl Med 1998;25:1429-34.

17. Avril N, Rosé CA, Schelling M, et al. Breast imaging with positron emission tomography and fluorine-18 fluorodeoxyglucose: use and limitations. J Clin Oncol 2000;18:3495-502.

18. Groheux D, Giacchetti S, Moretti JL, et al. Correlation of high 18F-FDG uptake to clinical, pathological and biological prognostic factors in breast cancer. Eur J Nucl Med Mol Imaging 2011;38:426-35.

19. Carkaci S, Macapinlac HA, Cristofanilli M, et al. Retrospective study of $18 \mathrm{~F}-\mathrm{FDG}$ PET/CT in the diagnosis of inflammatory breast cancer: preliminary data. J Nucl Med 2009;50:231-8.

20. van der Hoeven JJ, Krak NC, Hoekstra OS, et al. 18F-2fluoro-2-deoxy-d-glucose positron emission tomography in staging of locally advanced breast cancer. J Clin Oncol 2004;22:1253-9.

21. Alberini JL, Lerebours F, Wartski M, et al. 18F-fluorodeoxyglucose positron emission tomography/ computed tomography (FDG-PET/CT) imaging in the staging and prognosis of inflammatory breast cancer. Cancer 2009;115:5038-47.

22. Bakheet SM, Powe J, Kandil A, et al. F-18 FDG uptake in breast infection and inflammation. Clin Nucl Med 2000;25:100-3.

23. Yamauchi H, Woodward WA, Valero V, et al. Inflammatory breast cancer: what we know and what we need to learn. Oncologist 2012;17:891-9.

24. Carkaci S, Adrada BE, Rohren E, et al. Semiquantitative analysis of maximum standardized uptake values of regional lymph nodes in inflammatory breast cancer: is there a reliable threshold for differentiating benign from malignant? Acad Radiol 2012;19:535-41.

25. Palangie T, Mosseri V, Mihura J, et al. Prognostic factors in inflammatory breast cancer and therapeutic implications. Eur J Cancer 1994;30A:921-7. 
26. Chevallier B, Asselain B, Kunlin A, et al. Inflammatory breast cancer. Determination of prognostic factors by univariate and multivariate analysis. Cancer 1987;60:897902.

27. Anderson WF, Schairer C, Chen BE, et al. Epidemiology of inflammatory breast cancer (IBC). Breast Dis 20052006;22:9-23.

28. Ueno NT, Espinosa Fernandez JR, Cristofanilli M, et al. International Consensus on the Clinical Management of Inflammatory Breast Cancer from the Morgan Welch Inflammatory Breast Cancer Research Program 10th Anniversary Conference. J Cancer 2018;9:1437-47.

29. Takiar V, Akay CL, Stauder MC, et al. Predictors of durable no evidence of disease status in de novo metastatic inflammatory breast cancer patients treated with neoadjuvant chemotherapy and post-mastectomy radiation. Springerplus 2014;3:166.

30. Akay CL, Ueno NT, Chisholm GB, et al. Primary tumor resection as a component of multimodality treatment may improve local control and survival in patients with stage IV inflammatory breast cancer. Cancer 2014;120:1319-28.

31. Groheux D, Giacchetti S, Delord M, et al. 18F-FDG PET/CT in staging patients with locally advanced or inflammatory breast cancer: comparison to conventional staging. J Nucl Med 2013;54:5-11.

32. Kennecke H, Yerushalmi R, Woods R, et al. Metastatic behavior of breast cancer subtypes. J Clin Oncol 2010;28:3271-7.

33. Rosen EL, Eubank WB, Mankoff DA. FDG PET, PET/ CT, and breast cancer imaging. Radiographics 2007;27 Suppl 1:S215-29.

34. Tatsumi M, Cohade C, Mourtzikos KA, et al. Initial experience with FDG-PET/CT in the evaluation of breast cancer. Eur J Nucl Med Mol Imaging 2006;33:254-62.

35. Moon DH, Maddahi J, Silverman DH, et al. Accuracy of whole-body fluorine-18-FDG PET for the detection of recurrent or metastatic breast carcinoma. J Nucl Med 1998;39:431-5.

36. Cook GJ, Houston S, Rubens R, et al. Detection of bone metastases in breast cancer by 18 FDG PET: differing

Cite this article as: Patel MM, Le-Petross HT. Baseline FDG PET-CT imaging is necessary for newly diagnosed inflammatory breast cancer patients: a narrative review. Chin Clin Oncol 2021;10(6):56. doi: 10.21037/cco-21-82 metabolic activity in osteoblastic and osteolytic lesions. J Clin Oncol 1998;16:3375-9.

37. Even-Sapir E, Metser U, Flusser G, et al. Assessment of malignant skeletal disease: initial experience with 18F-fluoride PET/CT and comparison between 18F-fluoride PET and 18F-fluoride PET/CT. J Nucl Med 2004;45:272-8.

38. Isasi CR, Moadel RM, Blaufox MD. A meta-analysis of FDG-PET for the evaluation of breast cancer recurrence and metastases. Breast Cancer Res Treat 2005;90:105-12.

39. Kim TS, Moon WK, Lee DS, et al. Fluorodeoxyglucose positron emission tomography for detection of recurrent or metastatic breast cancer. World J Surg 2001;25:829-34.

40. Nakai T, Okuyama C, Kubota T, et al. Pitfalls of FDGPET for the diagnosis of osteoblastic bone metastases in patients with breast cancer. Eur J Nucl Med Mol Imaging 2005;32:1253-8.

41. Fuster D, Duch J, Paredes P, et al. Preoperative staging of large primary breast cancer with $[18 \mathrm{~F}]$ fluorodeoxyglucose positron emission tomography/computed tomography compared with conventional imaging procedures. J Clin Oncol 2008;26:4746-51.

42. Niikura N, Costelloe CM, Madewell JE, et al. FDG-PET/ CT compared with conventional imaging in the detection of distant metastases of primary breast cancer. Oncologist 2011;16:1111-9.

43. Yang WT, Le-Petross HT, Macapinlac H, et al. Inflammatory breast cancer: PET/CT, MRI, mammography, and sonography findings. Breast Cancer Res Treat 2008;109:417-26.

44. Walker GV, Niikura N, Yang W, et al. Pretreatment staging positron emission tomography/computed tomography in patients with inflammatory breast cancer influences radiation treatment field designs. Int J Radiat Oncol Biol Phys 2012;83:1381-6.

45. Niikura N, Liu J, Costelloe CM, et al. Initial staging impact of fluorodeoxyglucose positron emission tomography/computed tomography in locally advanced breast cancer. Oncologist 2011;16:772-82. 